\title{
Untersuchungen an der Heringslarvenbevölkerung der Innenjade ${ }^{1}$ ]
}

\author{
Von A. Bückmann und G. Hempel \\ Aus dem Max-Planck-Institut für Meeresbiologie in Wilhelmshaven und der \\ Biologischen Anstalt Helgoland in Hamburg \\ (Mit 5 Abbildungen und 5 Tabellen)
}

Im Plankton der südlichen Nordsee werden während des Winters fast stets Heringslarven in räumlich und zeitlich wechselnder Anzahl beobachtet. Im Frühjahr verschwinden diese Larven aus der offenen See, statt dessen treten dann große Larvenansammlungen unter den deutschen Küsten und in den Ästuaren auf. Bei Untersuchungen in der offenen See konnte BückmanN (1950) bezüglich der Längenzusammensetzung und geographischen Verbreitung unterschiedene Larvenvölker auf die einzelnen Laichakte im Herbst und Winter in der westlichen und südlichen Nordsee zurückführen. Nach Eindringen der Larven in das Küstenwasser gelang eine herkunftsmäßige Unterscheiding der Larvenschwärme bei diesen Vorkriegsuntersuchungen nicht mehr. Erst durch regelmäßige Beobachtungsfahrten in einem beschränkten Untersuchungsgebiet, der Innenjade, wurde nach dem Kriege eine solche Trennung möglich, über die hier berichtet werden soll. Darüber hinaus bot das Material die Möglichkeit, die Variation der Wirbelzahl eingehend zu betrachten.

In den Jahren 1949-1953 wurden auf einem NNW-gerichteten Schnitt von Wilhelmshaven bis zur Außenjade auf 5-7 Stationen nach Möglichkeit mindestens einmal monatlich Larvenfänge mit Petersens Jungfischtrawl ausgeführt. Sie lieferten folgendes Bild vom Aufenthalt der Heringsbrut im Jadegebiet:

Im Februar dringen Heringslarven in wechselnder Anzahl in die Jade ein, in den darauffolgenden Monaten schwankt die Bestandsdichte sehr stark. Im Mai verlassen die Larven das Jadegebiet, die Mehrzahl von ihnen steht dann in der Metamorphose. Das schubweise Eindringen größerer Mengen von Larven („Larvenwellen") in die Jade erfolgt unregelmäßig. Über den Aufenthalt des einzelnen Individiums im Untersuchungsgebiet wissen wir nichts. Die Schwankungen im Gesamtertrag der einzelnen Untersuchungsfahrten und zwischen den Fangerträgen aus den einzelnen Gebieten in und vor der Jade weisen auf einen regen Austausch hin.

1) Die Untersuchungen wurden $z, T$. mit Mitteln der Deutschen Wissenschaftlichen Kommission für Meeresforschung durchgeführt und durch ein Stipendium der Deutschen Forschungsgemeinschaft gefördert. Eine Zusammenfassung der Ergebnisse lag dem Special Meeting on Herring Races, Internationaler Rat für Meeresforschung, Kopenhagen 1956 vor (BǘcKMANN und HeMpec 1956), die dort gegebenen Zahlenwerte erfuhren einige Korrekturen auf Grund z. T. neuer, genauerer Unterlagen. - Karte des Untersuchungsgebietes bei GrLlbricht 1956 . 
Helgol. Wiss. Meeresunters. 6, 1 : Bückmann u. Hempel

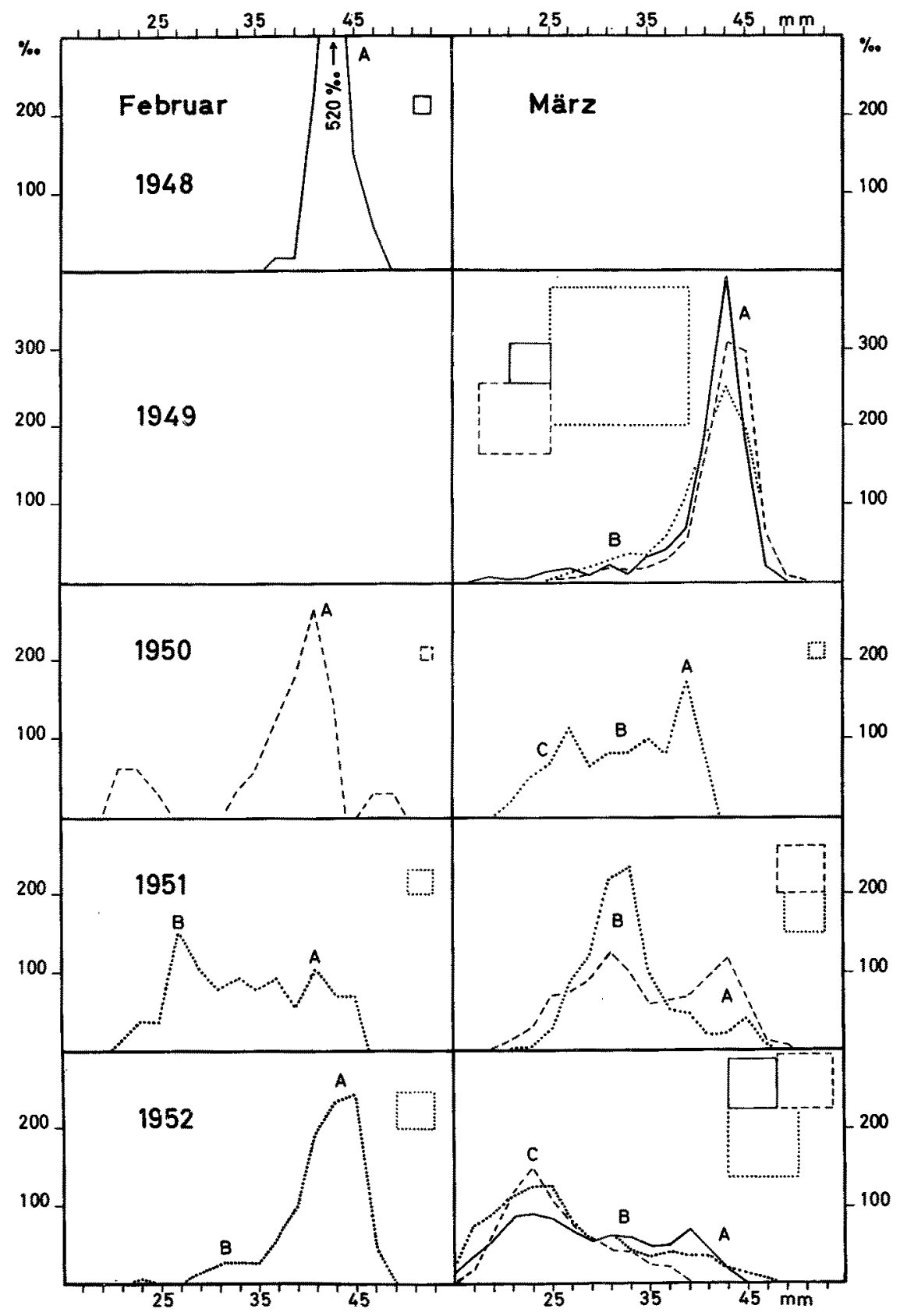

Abb. 1 (a)

Abb. 1. Heringslarven: Längenverteilung der Jahrgänge 1948-52 während des darauffolgenden Frühjahrs in der Jade. Monate Februar, März (a) und April, Mai (b).

Vermutliche Herkunft der Larvengruppen: $\mathrm{A}=$ mittlere Nordsee, $\mathrm{B}=$ Doggerbankgebiet, $\mathrm{C}=$ Downsgebiet (November/Dezember), $\mathrm{D}=$ Downsgebiet - östl. Kanal? (Januar).

- Verteilung Anfang des Monats,

_ _ _ - Verteilung Mitte des Monats,

........ Verteilung Ende des Monats. 
Helgol. Wiss. Meeresunters. 6, 1 : Bückmann u. Hempel

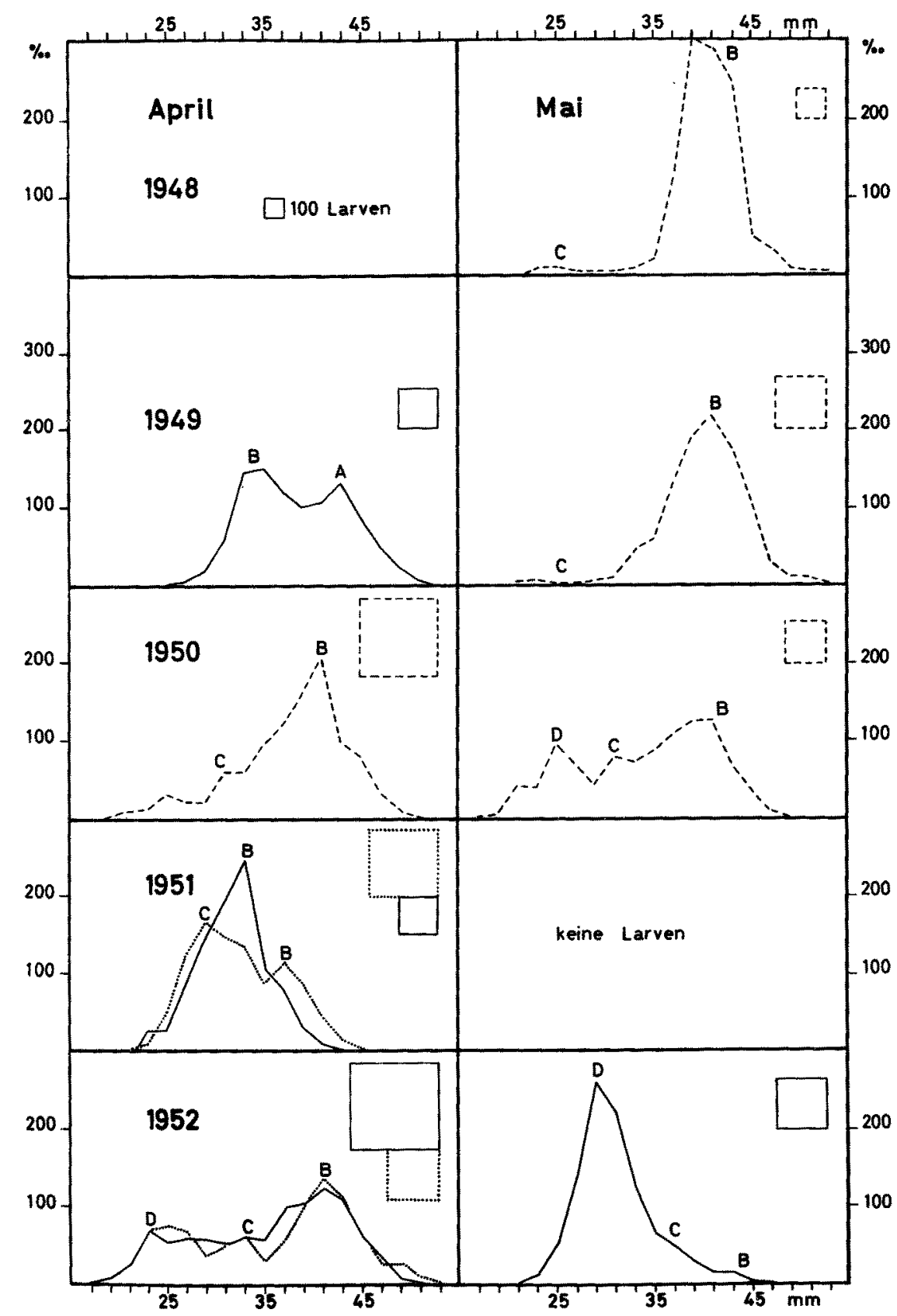

Abb. 1 (b) 


\section{Die Körperlänge}

Die Abbildung 1 gibt für die einzelnen Untersuchungsfahrten die mittlere Längenzusammensetzung in den Heringslarvenproben wieder. Gelegentlich wurden mehrere kleine Fangproben gemeinsam dargestellt, wenn sie in ihrer Zusammensetzung annähernd übereinstimmten. Wie bei den früheren Untersuchungen maßen wir stets die Totallänge der Larve. Um eine Úbersicht zu erzielen, sind die Gipfel in den einzelnen Längenverteilungen in der Reihenfolge ihres Auftretens mit Buchstaben bezeichnet. Im weiteren Verlauf der Betrachtungen konnten diese Gipfel bestimmten Larvengruppen verschiedener Herkunft zugeordnet werden.

Im Jahre 1949 (Jahrgang 1948) konnte nur im Februar und im Mai auf Heringslarven gefischt werden. Anfang Februar wurden im Eingang der Jade einige große Larven $(40-50 \mathrm{~mm}$, Gruppe A) festgestellt. - Mitte Mai herrschten Larven um $40 \mathrm{~mm}$ Körperlänge vor (Gruppe B). Einzelne Larven waren $z$ wischen 23 und $33 \mathrm{~mm}$ lang (Gruppe C).

Als Anfang März 1950 die erste "Welle" der Heringslarven des Jahrgangs 1949 in die Jade eindrang, war ihre Längenverteilung ähnlich der im Februar 1949 beobachteten. Neben die Gruppe sehr großer Larven (A) traten in den nächsten Wochen kleinere Tiere, die am 6. 4. den Hauptgipfel bei 33-35 mm (Gruppe B) bildeten. Es war wohl die gleiche Larvengruppe, die im Mittel um $40 \mathrm{~mm}$ lang am 10.5. in der Verteilung dominierte ${ }^{2}$ ), ihr scheinen Angehörige einer Gruppe mit viel niedrigerem Längenmittel vereinzelt beigemischt gewesen zu sein (Gruppe C).

1951 (Jahrgang 1950) wurden Mitte Februar nur wenige, meist große Larven (um $40 \mathrm{~mm}$, Gruppe A) gefangen. Erheblich kleinere Larven standen Ende Februar v o r der Jade (Gruppe B), hier fehlten die großen Larven. Erst im April wurden wieder größere Larvenmengen in der Jade angetroffen. Die Larven der Gruppe B waren Mitte April > $35 \mathrm{~mm}$ lang, ihre Längenverteilung überlagerte sich mit den letzten Larven der Gruppe A. Am 11. 5. war die Längenverteilung mehrgipfelig: die größeren Tiere um $40 \mathrm{~mm}$ gehörten wohl zur Gruppe B, daneben erschienen eine oder zwei neue Gruppen C/D mit einem Längenmittel von $25 \mathrm{~mm}$.

Die Verteilung der Heringslarven des Jahrgangs 1951 in See und vor der ostfriesischen Küste im Frühjahr 1952 wurde von BückMANN (1953) und Bückmann und Hempel (1953) geschildert. In der Jade konnten erst Mitte März größere Larvenansammlungen festgestellt werden: Neben der Gruppe großer Larven (A) zeigt die Längenverteilung einen starken Gipfel um $30 \mathrm{~mm}$ $(\text { Gruppe B })^{3}$ ). Während Mitte April nur vereinzelt Larven in und vor der Jade beobachtet wurden, traten Ende April neue Larvenschwärme auf. Die um 35 bis $40 \mathrm{~mm}$ langen Larven dürften der Gruppe B zugehören, daneben zeichnet

2) Es besteht ein Austausch von Heringslarven zwischen Außen- und Innenjade, der bei den kleineren Larven z. T. durch den unregelmäßigen Wasseraustausch (Gillbricht 1956) bewirkt wird. Die einzelnen in ihrer Längenverteilung unterschiedenen Gruppen stellen in der Jade keine abgeschlossenen Einheiten dar. Man kann daher nicht aus der Verschiebung der I.ängenmittel in den einzelnen Gruppen im Verlaufe der Zeit das mittlere Individualwachstum ablesen. Z. B. kommen von See Nachzügler an, die kleiner sind, als die bereits in der Jade lebenden Tiere der gleichen Gruppe.

3) Eine ähnliche Längenverteilung wie 1952 in der Jade fand sich im März 1955 im Küstenwasser der Deutschen Bucht, während um die gleiche Zeit die Innen-Jade fast frei von Larven war. 
sich ein starker Gipfel bei $28 \mathrm{~mm}$ (Gruppe G) ab. Im Mai waren fast alle Larven aus der Jade verschwunden.

Auch 1953 wurde die Verteilung der Heringslarven (Jahrgang 1952) in See durch Fahrten mit "Gauss" (BoHL 1954) und "Uthörn" untersucht. In der Jade wurden Ende Februar vor allem große Larven (43 mm, Gruppe A) gefangen. Eine stark durchmischte Bevölkerung trat im März auf. Einige sehr große Larven gehörten wahrscheinlich noch der Gruppe A an. Neben einem flachen Kurvenverlauf zwischen 27 und $37 \mathrm{~mm}$, der wahrscheinlich auf eine oder mehrere Larvengruppen (Gruppe B) zurückgeführt werden muß, bestand ein Gipfel bei 20-25 mm (Gruppe C), denen noch kleinere Tiere folgten. Mitte April waren die Larven der Gruppe B etwa $40 \mathrm{~mm}$ lang, die der Gruppen C/D $25 \mathrm{~mm}$ und größer, am 8. 5. erreichten diese Tiere im Mittel ca $30 \mathrm{~mm}$.

Vergleicht man die für die einzelnen Jahre unabhängig voneinander aufgestellten Gruppeneinteilungen, so ergeben sich auffallende Ubereinstimmungen: Zu Beginn der Saison bis etwa Ende März trat in allen Jahren eine Gruppe großer Larven auf. Gleichzeitig oder etwas später wurden Larven der Gruppe B beobachtet, die Ende März 30-35 mm lang waren und im Mai mit mehr als $40 \mathrm{~mm}$ kurz vor der Metamorphose standen. Die Gruppe C umfaßte auffallend kleine Larven. Es ist unwahrscheinlich, daß es sich hierbei um eine einzelne geschlossene Gruppe handelte. Eine Aufteilung in ..ältere" Downslarven und "jüngere", wie sie bei den Seeproben möglich ist, läßt sich aber bei den Jadefängen nicht mit Sicherheit durchführen. Die Unterscheidung in die Gruppen $\mathrm{C}$ und $\mathrm{D}$ in Abb. $1 \mathrm{~b}$ ist als hypothetisch zu betrachten. Aus den Untersuchungen in See (Bückmann 1950, 1953, Bückmann und Hempel 1953, BoHz 1954, Hempel 1957) und in der Jade ergibt sich insgesamt folgendes Bild der Zusammensetzung des Heringslarvenbestandes in der Deutschen Bucht und an der Ostfriesischen Küste:

1. Wahrscheinlich von den Laichplätzen der Mittleren Nordsee stammen die Larven des Bankherings, die um $40 \mathrm{~mm}$ lang im Februar im inneren Teil der deutschen Wattenmeere auftauchen.

2. Die Heringslarven aus dem Laichgebiet der Doggerbank haben im Januar Körperlängen um und wenig über $25 \mathrm{~mm}$ erreicht. Mit 30-35 mm Länge gelangen sie im März ins Wattenmeer, wo etwa im Mai die Metamorphose erfolgt.

3. Im Südteil der Flämischen Bucht schlüpfen Ende November bis Mitte Dezember Heringslarven, die im Januar um $12 \mathrm{~mm}$ lang sind. Im März in der Deutschen Bucht liegt ihre mittlere Länge von Jahr zu Jahr schwankend zwischen 20 und $25 \mathrm{~mm}$. Sie dringen - etwa 30-35 mm lang - im April bis Mai in die Jade ein. Ihrem Herkunftsgebiet entsprechend werden sie als „ältere Downslarven“ bezeichnet.

4. Eine weitere Gruppe erreicht im März 12-17 mm. Sie gelangt nicht in allen Jahren in die Deutsche Bucht. 1952 und 1955 wurden solche Larven nur im Südteil der Flämischen Bucht gefangen, sie dürften dem Laichen im Januar im östlichen Kanal entstammen (,jüngere Downslarven“).

1937 und 1953 wurde auch in der Deutschen Bucht diese Gruppe kleiner Larven nachgewiesen. Bei den widrigen Strömungen des Frühjahres 1953 können diese Larven aber im Gegensatz zu den Verhältnissen 1937 nicht vom Kanalausgang bis zum ostfriesischen Wattenmeer gelangt sein, viel- 
mehr muß nach BoHL (1954) in diesem Jahr ihr Herkunftsgebiet im Nordteil der Flämischen Bucht oder auf dem südlichen Austerngrund gelegen haben. Ein Winterlaichen von Heringen in diesem Gebiet ist bisher nicht bekannt.

Nur die Abkömmlinge der Sommerlaicher der Mittleren Nordsee und evtl. die Doggerbank-Larven kommen in einem so weit fortgeschrittenen Entwicklungsstadium in den Bereich unserer Küste, daß für sie ein aktives Einwandern in das Küstenwasser angenommen werden kann. Der dieser Wanderung zu den Freßgebieten zugrunde liegende Orientierungsmechanismus ist unbekannt. Die kleineren Doggerbank-Larven und die Downslarven erreichen das Küstenwasser auf einer früheren Entwicklungsstufe. Für sie kommt nur ein passiver Transport in Frage. Die von Dietrich (1953) vor Texel und später auch in der Deutschen Bucht festgestellten Vermischungsvorgänge an der Konvergenz geben die Möglichkeit passiver Verfrachtung in das Küstenwasser, sofern die Heringslarven außen in Bodennähe stehen (BüCKMANN 1953). Ein solcher Transport ist an bestimmte Strom- und Gezeitenverhältnisse gebunden, die nur in einzelnen Küstengebieten gegeben sind. $\mathrm{Ob}$ noch anderen Transportmechanismen Bedeutung zukommt, ist unbekannt. Mit den Möglichkeiten der Ausbreitung des Planktons innerhalb des Küstenwassers des Jadegebietes befaßte sich GILLBRICHT (1956).

\section{Die Wirbelzahl}

Die mittlere Wirbelzahl wird von den meisten Untersuchern als eines der wichtigsten Hilfsmittel zur Unterscheidung von Heringsproben verschiedener Herkunft verwendet. Die Wirbelzählungen von TEsch (1937) und BüCKMANN (1950) an Heringslarven hatten keine Handhabe zur sicheren Trennung verschiedener Larvengruppen geliefert. HAMDORF (1956) hat ferner gezeigt, daß entgegen unserer früheren Annahme auch der Verknöcherungszustand der Wirbelsäule kein brauchbares Unterscheidungsmerkmal ist.

Aufgabe der hier geschilderten Untersuchungen war es, die früher mitgeteilten Befunde (BücKMANN 1950) an neuem Material zu überprüfen, weitere Kenntnisse über die Faktoren, welche die Wirbelzahl beeinflussen, zu sammeln und so einem sicheren Urteil über die Brauchbarkeit der Wirbelzahl als „Rassenmerkmal“ näher zu kommen. Zur Bestimmung der Wirbelzahl von 4964 Heringslarven wurde die von Bückmann (1950) beschriebene Methode verwendet. Bei der Betrachtung der einzelnen Jahrgänge ist zu beachten, daß jeweils dasjenige Jahr genannt wurde, in dem die Masse der Larven (Herkunft: Mittlere Nordsee, Doggerbankgebiet, südliche Flämische Bucht) schlüpfte, ohne Rücksicht darauf, daß etwa beigemischte Kanallarven aus dem Januar des folgenden Jahres (und damit des Untersuchungsjahres der Larven) stammen können.

\section{a) Die Variation der Wirbelzahl}

BücKMANN (1950) hatte für das Vorkriegsmaterial nachgewiesen, daß die Verteilungskurve der Wirbelsummen bei den Heringslarven von der Zufallskurve abweicht, und zwar hatte er eine starke Ubergipfeligkeit (positiver Exzeß) der empirischen Verteilungskurve festgestellt. Die Verteilungskurve ist aber nicht nur zu spitz sondern auch asymmetrisch. An Stelle des hier nicht voll anwendbaren $\chi^{2}$-Verfahrens wurde die Signifikanz dieser Abweichungen 
von der Normalverteilung erneut auf dem u. a. bei Goulden (1952) angegebenen Weg der Berechnung der 3. und 4. Momente bestimmt. Es ergibt sich mit wenigen Ausnahmen, die vorwiegend auf zu geringen Materialumfang zurückzuführen sind, eine statistische Sicherung für positiven Exzeß $\left(\mathrm{g}_{2}\right)$ und eine positive Schiefe $\left(g_{1}\right)$ der Verteilungen von Vert. S. in den einzelnen Jahrgängen (Tab. 1). Ein positiver Exzeß bedeutet nicht allein eine im Vergleich zur Normalverteilung zu große Häufigkeit der dem Mittelwert benachbarten Varianten ( 56 und 57 Wirbel) bei Mangel an mäßigen Abweichungen ( 55 und 58 Wirbel), sondern auch ein zu starkes Vorkommen von Extremvarianten ( 54 und 59 Wirbel). Die positiven Werte für die Schiefe weisen auf ein Überwiegen der positiven Extremvarianten und im Vergleich zur Lage des Mittelwertes zu häufiges Auftreten der schwach negativen Varianten hin. Nur bei dem Jahrgang 1951 lagen die Verhältnisse umgekehrt, hier war die Schiefe negativ und es fehlte der Exzeß. Eine Zusammenfassung der nicht voll gesicherten $g_{1}$ und ge beim Jahrgang 1931 ergibt auch für ihn eine signifikante Gesamtabweichung von der Normalverteilung.

Tabelle I

Mittlere Wirbelzahl der Heringslarven und die Abweichungen von der Normalverteilung

\begin{tabular}{lrcrcrr} 
Jahrg. & $\mathbf{n}$ & Vert. S. & $\mathrm{g} 1$ & $\mathbf{P}_{\mathrm{g} 1}$ & $\mathrm{~g} 2$ & $\mathbf{P}_{\mathrm{g} 2}$ \\
\hline 1928 & 985 & 56.4994 & $-0,0914$ & $<0,05$ & $+0,5691$ & $<0,01$ \\
1929 & 2543 & 56.4994 & $+0,1900$ & $<0,01$ & $+0,3538$ & $<0,01$ \\
1930 & 2191 & 56.4409 & $+0,2980$ & $<0,01$ & $+0,3688$ & $<0,01$ \\
1931 & 2241 & 56.4114 & $+0,1047$ & $<0,05$ & $+0,2443$ & $<0,05$ \\
1932 & 1653 & 56.4386 & $+0,1768$ & $<0,01$ & $+0,8812$ & $<0,01$ \\
1933 & 925 & 56.5978 & $+0,1627$ & $<0,05$ & $+0,2393$ & $>0,05$ \\
1934 & 372 & 56.4328 & $-0,0691$ & $>0,05$ & $-0,2980$ & $>0,05$ \\
& & & & & & $<0,01$ \\
1948 & 277 & 56.5307 & $+0,6169$ & $<0,01$ & $+2,5295$ & $<0,01$ \\
1949 & 993 & 56.5368 & $-0,0775$ & $>0,05$ & $+0,7366$ & $>0,05$ \\
1950 & 902 & 56.5466 & $-0,3877$ & $<0,01$ & $-0,1112$ & $>0,05$ \\
1951 & 1038 & 56.5106 & $-0,1321$ & $\sim 0,05$ & $+0,1565$ & $\sim 0,03$ \\
1952 & 1754 & 56.5125 & $-0,0110$ & $>0,05$ & $+0,2565$ & $\sim 05$
\end{tabular}

Die hier beschriebenen Abweichungen von der Normalverteilung haben nicht nur erhebliches theoretisches Interesse bei der Diskussion von Veränderungen in der Zusammensetzung der Larvenbevölkerung. Sie sind auch methodisch von Bedeutung. Die in der Fischereibiologie gebräuchlichen statistischen Verfahren fußen auf der Annahme, daß die Varianten innerhalb der untersuchten Probe annähernd "normal" verteilt sind. Nur unter dieser Bedingung liefern die üblichen Angaben vom Mittelwert, Probenumfang und Streuung ein Bild von der Verteilung der Varianten, das sich - je größer die Probe, desto enger - mit der tatsächlichen Verteilungskurve deckt. Besteht diese Ubereinstimmung aber wie in unserm Falle nicht, so sind Berechnungen auf der Grundlage der genannten statistischen Größen streng genommen unzulässig und können zu Fehlschlüssen führen. An die Stelle der üblichen statistischen Methoden müssen solche treten, die gegen Abweichungen von der Normalverteilung weniger empfindlich sind, z. B. das Arbeiten im logarithmischen System.

Bei der hier vorliegenden Darstellung haben wir die herkömmliche Verwendung. des Mittelwertes beibehalten, um Vergleiche mit älteren Angaben 


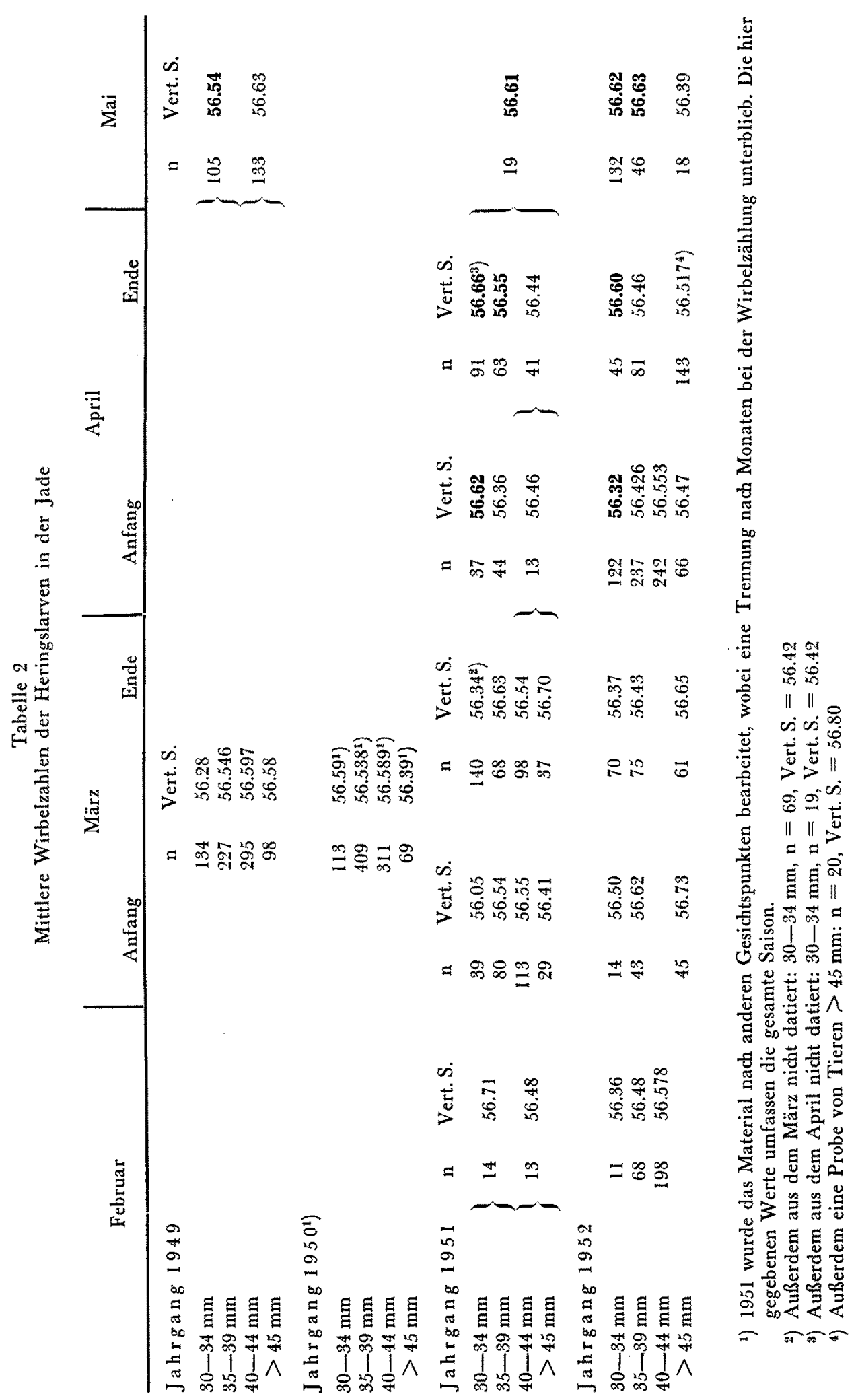


zu ermöglichen. Bei Folgerungen aus dem Vergleich der Vert.S. verschiedener Jahre oder Jahresgruppen wird aber - sofern die Differenzen gering sind Vorsicht geboten sein.

\section{b) Wirbelzahl und Körpergröße}

Bei den 1929 bis 1935 in der inneren Deutschen Bucht und in den Ästuaren gefangenen Heringslarven hatte BüCKMANN (1950) eine positive Korrelation von Wirbelzahl und Körpergröße nachgewiesen. Ähnliche Beobachtungen wurden bei Clupea pallasii an Larven. (Mc Hugr 1942) und adulten Tieren (Thompson 1917, zit. nach Tester 1937, Tester 1937 im Gegensatz zu Tester 1949) gemacht.

In Tab. 2 wurde die Wirbelzahl der in den Jahren 1950-1953 untersuchten Heringslarvenproben, in 5-mm-Klassen gruppiert, dargestellt $\left.\mathrm{t}^{4}\right)$. Wie aus der Tabelle hervorgeht, sind Beziehungen zwischen mittlerer Körpergröße und Vert.S. nur bei einem Teil des Jadematerials offensichtlich. Sie lassen sich 1950 und in der ersten Saisonhälfte 1953 ablesen, nicht dagegen bei dem Material der Jahre 1951, 1952 und Ende April-Anfang Mai 1953. Eine Zusammenfassung des gesamten Nachkriegsmaterials läßt zwar den allgemeinen Anstieg erkennen:

\begin{tabular}{ccc} 
Körperlänge & Vert.S. & $\mathrm{n}$ \\
\hline $30-34 \mathrm{~mm}$ & 56,446 & 1050 \\
$35-39 \mathrm{~mm}$ & 56,513 & 1538 \\
$>40 \mathrm{~mm}$ & 56,564 & 2020
\end{tabular}

Die Jahresmittel der einzelnen 5-mm-Gruppen von 1952 und 1953 zeigt Abb. 2. Gruppiert man aber nach Monaten (Tab. 3), wie es Bückmann (1950, Tab. 19 - S. 199) für die Vorkriegsproben durchgeführt hatte, so ist das Bild weniger einheitlich. Für März zeigt sich ein gleichmäßiger Anstieg, im April weisen die kleinsten Larven relativ hohe Werte auf, während Vert.S. bei den 35-39 mm langen Tieren niedriger ist als im März. Im Mai lassen sich keine Beziehungen zwischen Körpergröße und Wirbelzahl feststellen.

Aus Tab. 1 und 3 geht hervor, daß zwischen den in den Vorkriegsjahren ermittelten Wirbelsummen und den Nachkriegswerten auffallende Unterschiede bestehen. Eine statistische Überprüfung ergab, daß die Mittelwerte von Vert.S. der Jahre 1929-34 gesichert unter denen der Nachkriegsjahre liegen. In gewissem Umfange dürften dafür Unterschiede in der mittleren Körperlänge der in den beiden Zeiträumen untersuchten Larven von Bedeutung sein. In den Vorkriegsjahren setzte sich das Material zu einem erheblichen Teil aus relativ kleinen Larven, die das Küstengebiet noch nicht erreicht hatten, zusammen. Tab. 3 zeigt diese Unterschiede in der Längenzusammensetzung. Die Vert.S.-Differenzen $z$ wischen den beiden Untersuchungszeiträumen bei den einzelnen Längengruppen sind etwas geringer als bei den Gesamtsummen von Tab. 1, und eine feinere Längenaufgliederung würde wahrscheinlich diese Unterschiede noch weiter ausgleichen. Dennoch scheint es nicht gerechtfertigt. die höheren Vert.S.-Werte der Nachkriegsjahre einzig durch die andere

4) 1949 wurden im Februar (Vert.S. 56,62, $\mathrm{n}=68$ ) und Mai (Vert.S. $56,50, \mathrm{n}=209$ ) Wirbelzählungen durchgeführt, ohne daß dabei eine Trennung nach Längengruppen erfolgte. In beiden Monaten handelte es sich um große Larven $(>35 \mathrm{~mm})$. 
Helgol. Wiss. Meeresunters. 6, 1 : Bückmann u. Hempel

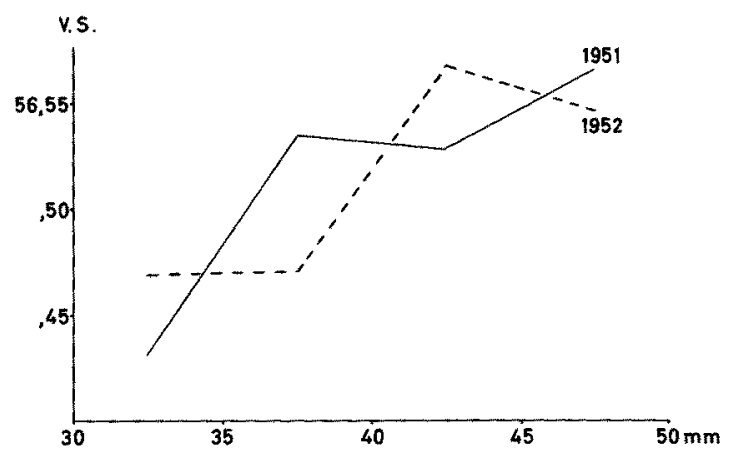

Abb. 2. Beziehung zwischen Wirbelzahl und Körpergröße bei Hexingslarven in der Jade. Mittlere Wirbelzahl von 5-mm-Gruppen der Jahrgänge 1951 und 1952.

v.s.

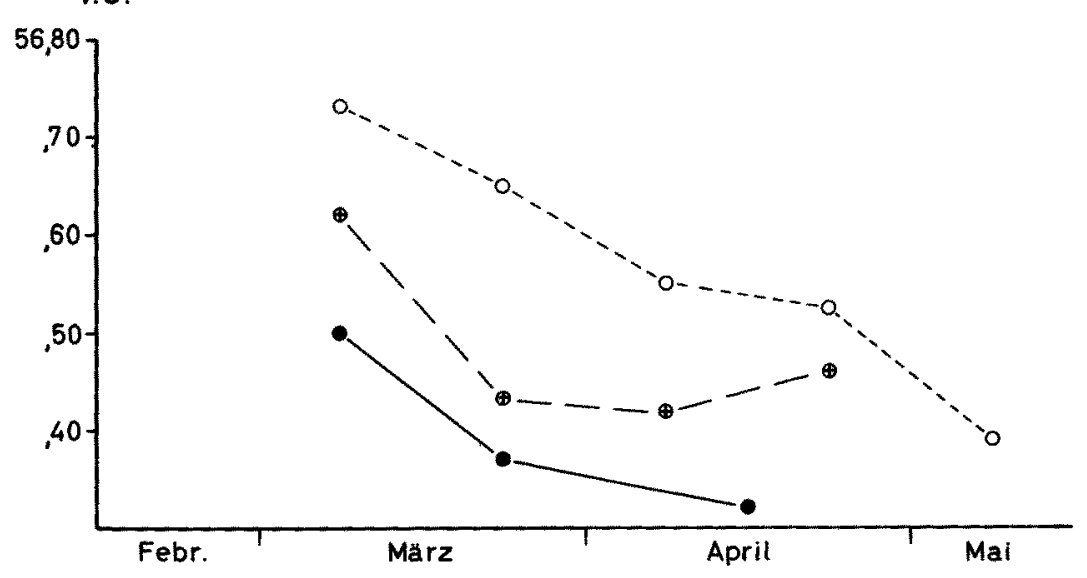

Abb. 3. Beziehungen zwischen Wirbelzahl und Körperlänge (relativ zur Längenverteilung in der Larvengruppe) in einer isolierten Larvengruppe (Doggerbank-Larven Jahrg. 1952). Abnahme der mittleren Wirbelzahl von 5-mm-Gruppen im Verlauf des Frühjahrs 1953

๑ि- $30-34 \mathrm{~mm}$

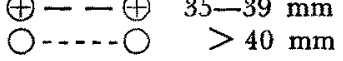


Tabelle 3

Mittlere Wirbelzahlen von Heringslarven der Jahrgänge 1928-34 und 1949-52

\begin{tabular}{|c|c|c|c|c|c|}
\hline & & & & & \\
\hline & $\mathrm{mm}$ & $\mathbf{n}$ & Vert. S. & $\mathbf{n}$ & Vert. S. \\
\hline März & $30-34$ & 1199 & 56.414 & 466 & 56.320 \\
\hline & $35-39$ & 2080 & 56.509 & 493 & 56.547 \\
\hline & $>40$ & 1075 & 56.547 & 751 & 56.595 \\
\hline April & $30-34$ & 1270 & 56.409 & 314 & 56.500 \\
\hline & $35-39$ & 2122 & 56.416 & 425 & 56.446 \\
\hline & $>40$ & 1164 & 56.476 & 525 & 56.530 \\
\hline Mai & $30-34$ & 449 & 56.494 & 132 & 56.62 \\
\hline & $35-39$ & 701 & 56.509 & 130 & 56.55 \\
\hline & $>40$ & 544 & 56.526 & 166 & 56.60 \\
\hline
\end{tabular}

*) Zusammengefaßt aus Tab. 19 bei Bückmann (1950, S. 199).

Längenzusammensetzung der Proben erklären zu wollen. Für die adulten Nordseeheringe ist eine deutliche Änderung der Wirbelzahl gegenüber den Vorkriegsjahren nicht festgestellt worden.

c) Wirbelzahl und Wachstumsgeschwindigkeit

Neben den Beziehungen zwischen Wirbelzahl und Körpergröße scheinen auch solche zwischen Wirbelzahl und Wachstumsgeschwindigkeit der Larven zu bestehen. Für Jahrgang 1952 wurden diejenigen Längengruppen getrennt betrachtet, die sich vorwiegend aus Doggerbank-Larven (Gruppe B) zusammensetzen. Im Februar 1953 besaßen nur einige der schnellwüchsigen Larven (hier .. Vorwüchser" genannt) dieser Gruppe eine hinlänglich verknöcherte Wirbelsäule, im März erreichten die Vorwüchser und ein Teil der normalwüchsigen Tiere eine Länge $>30 \mathrm{~mm}$, und im April traten auch die langsamwüchsigen Varianten (hier "Nachwüchser") der Doggerbank-Larven in diese Längengruppe. Läge nur eine Korrelation zwischen Wirbelzahl und Körperlänge vor, müßte während der ganzen Beobachtungszeit die Wirbelzahl der einzelnen Längengruppen konstant bleiben. Wie Abb. 3 zeigt, ist das aber nicht der Fall. In der Zeit von Anfang März bis, Ende April sinkt bei den Längengruppen $30 / 35$ bis $40 / 44 \mathrm{~mm}$ die mittlere Wirbelzahl ${ }^{5}$ ). Demnach wird eine bestimmte Körperlänge mit fortschreitender Jahreszeit von Larven mit immer niedrigerer Wirbelzahl erreicht. Die Vorwüchser unter den Doggerbank-Larven wiesen höhere Wirbelzahlen auf als die in die entsprechende Längengruppe später nachrückenden Normal- und Nachwüchser. Dabei liegt die Wirbelsumme bei den Vorwüchsern erheblich über dem für Doggerbank-Heringe zu erwartenden Wert, die der Nachwüchser darunter. Dies scheint nicht allein für die Doggerbank-Larven zu gelten, sondern allgemeinere Gültigkeit zu haben. HAMDORF (1954) fand bei Untersuchung des Larvenmaterials von Jahrgang 1952, daß die positiven Varianten einer unimodalen Längenverteilung relativ hohe Wirbelzahlen haben, bzw. bei Zweigipfeligkeit der Kurven jeweils die

5) Im Jahr 1952 ist die Trennung der Doggerbank-Larven von den übrigen Larvengruppen durch Populationsüberschneidungen innerhalb der betrachteten Längengruppen nicht so klar durchzuführen. Die Auftragung der Vert.S. der einzelnen Längengruppen gibt daher für das Jahr 1952 kein einheitliches Bild. 
größten Tiere der beiden Larvengruppen. Unter Verwendung der Zahlenwerte von HAMDORF wurden für drei Termine 1953 die mittlere Wirbelzahl von 2-mm-Gruppen aufgetragen (Abb. 4) und die Längenverteilung in den einzig für die Wirbeluntersuchung in Frage kommenden Längengruppen $>30 \mathrm{~mm}$ ähnlich wie in Abb. 1 zum Vergleich eingezeichnet:

Am 24. 2. bestand die Probe fast ausschließlich aus Abkömmlingen des Laichens in der mittleren Nordsee. In dem hier untersuchten Längenintervall $>30 \mathrm{~mm}$ herrschten am 19. 2. und 9. 4. als kleine Larven die Vorwüchser der Downslarven vor, die größeren Tiere stammten von der Doggerbank, die größten dürften teils Vorwüchser gleicher Herkunft, teils Nachwüchser der Larven aus der mittleren Nordsee sein. Bei der isolierten Larvengruppe der Februar-Probe (Abb. 4 a) können wir mit einem gleichmäßigen Anstieg der Wirbelzahl von den kleinen Nachwüchsern bis zu den großen Vorwüchsern rechnen. In den beiden späteren Proben (Abb. 4 b u. c) ist ein hoher Wert für die kleinen vorwüchsigen Downslarven, niedrigere Werte in der mittleren Längengruppe (Nach- und Normalwüchser vom Dogger) und hohe Werte für die um $40 \mathrm{~mm}$ langen Vorwüchser zu erwarten. Für die allergrößten Tiere sind keine sicheren Vorhersagen zu machen, da hier wahrscheinlich ein Gemisch von Larven verschiedener Herkunft vorliegt. Aus den Abbildungen geht eine gute Übereinstimmung der empirischen Werte mit unseren Erwartungerı hervor.

Mc Hugh (1942) fand innerhalb von Proben, die nur Abkömmlinge eines einzelnen Laichaktes umfaßten, eine Covariation zwischen Körpergröße und Wirbelzahl der Larven von Clupea pallasii. Er nimmt daher ebenfalls eine Verknüpfung mit der Entwicklungsgeschwindigkeit an.

In neuen Untersuchungen soll u. a. nochmals die Beziehung zwischen der Wirbelzahl und der Wachstumsgeschwindigkeit geprüft werden. Vor allem soll möglichst die Gruppe vorwüchsiger Doggerbank-Larven während des ganzen Frühjahrs beobachtet werden. Wenn lediglich eine Beziehung der Wirbelzahl zur Wachstumsgeschwindigkeit, nicht aber zur absoluten Körpergröße besteht (s. u. „Selektionshypothese“, S. 64 f.), wäre eine konstante Wirbelzahl in dieser Wachstumsgruppe unabhängig von Alter und Länge zu erwarten. Das bisher vorliegende Material reicht zur Entscheidung dieser Frage nicht aus.

\section{d) Wirbelzahl und Herkunft der Larven}

Bei den Vorkriegsuntersuchungen waren für die kleinsten und in der Jahreszeit am spätesten auftretenden Larven, unter denen man die Downslarven vermuten mußte, keine höheren Wirbelzahlen festgestellt worden. An dem hier vorgelegten Material fällt aber auf, daß diejenigen Larven, die wir auf Grund ihrer Körperlänge und ihres Ankunftstermins im Küstenwasser als Downslarven bezeichneten, hohe mittlere Wirbelzahlen (mit Ausnahme der Längengruppe 30-34 mm, Anfang April 1953) bei geringer mittlerer Körpergröße aufweisen. Die Längengruppen, die sich wahrscheinlich vorwiegend aus Downslarven zusammensetzen, wurden in Tab. 2 durch Fettdruck hervorgehoben. Es läßt sich nicht entscheiden, ob dieser Larvengruppe insgesamt eine höhere Wirbelzahl zukommt, sei es, daß diese entsprechend der höheren Werte bei den Elterntieren auf den südlichen Laichgründen genetisch bedingt ist oder daß die niedrigere Wassertemperatur während der frühwinterlichen Inkubationszeit und dem frühen Larvenleben steigernd auf die Wirbelausbildung wirkte. Es handelt sich bei den hier untersuchten Downslarven fast ausschließlich um Vorwüchser einer Larvengruppe, deren Gros nur selten in der Jade bis zur vollständigen Ausbildung der Wirbelsäule heranwächst. So 


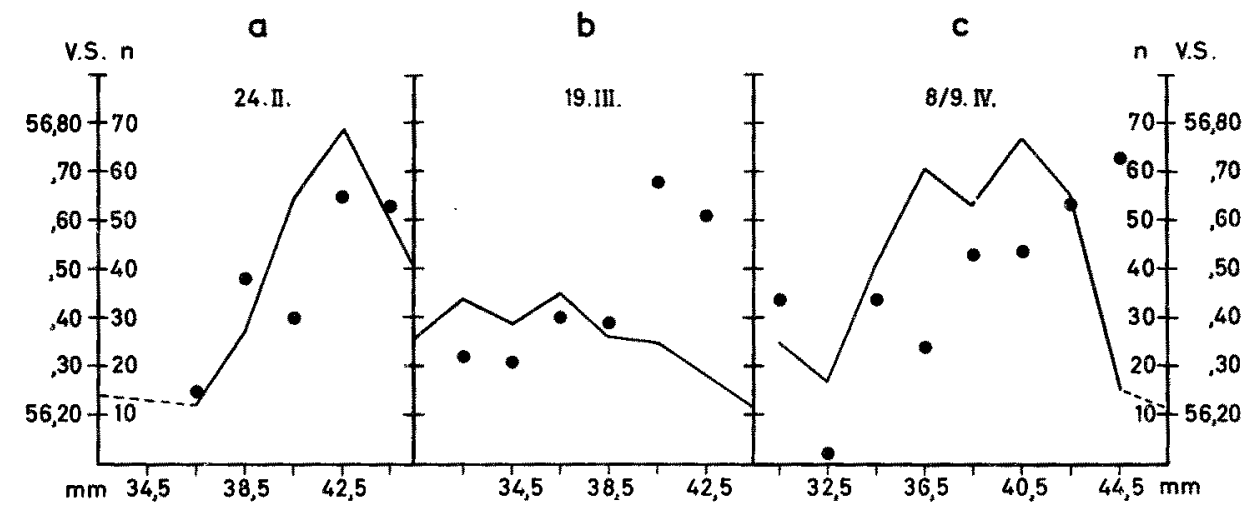

Abb. 4. Korrelation zwischen Wirbelzahl und Körpergröße (relativ zur Längenverteilung in der Larvengruppe).

Längenverteilung der Larven mit verknöcherter Wirbelsäule $(>30 \mathrm{~mm})$ (Anzahl der Tiere - $\mathrm{n}$ - in den einzelnen 2-mm-Gruppen)

- Mittlere Wirbelzahlen der einzelnen 2-mm-Gruppen, Frühjahr 1953

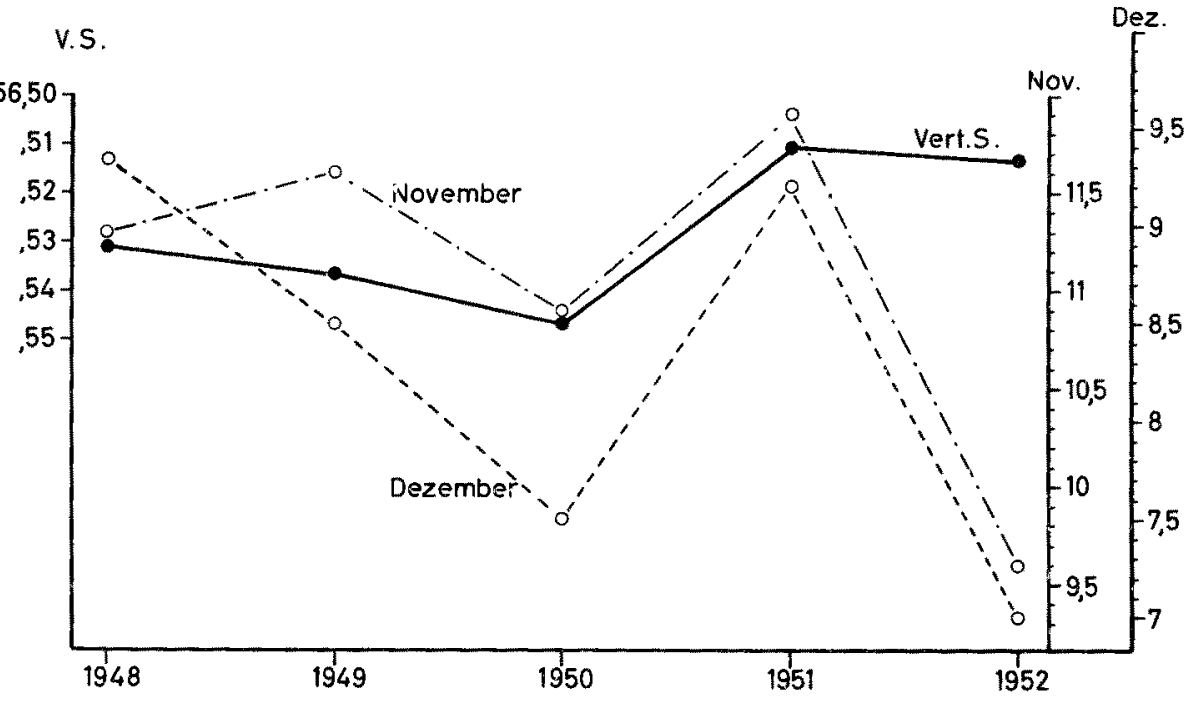

Abb. 5. Negative Korrelation zwischen Wirbelzahl und winterlicher Oberflächentemperatur bei Feuerschiff "S 2".

Wirbelzahl

_._.- mittlere Wassertemperatur im November 
muß die Möglichkeit offenbleiben, daß die Wirbelzahlen hoch liegen, weil es sich um Vorwüchser handelt, die uns unbekannte mittlere Wirbelzahl der g e samten Larvengruppe aber die der Doggerbank-Larven nicht übertrifft.

\section{e) Die Wirbelzahl einjähriger Heringe}

In jedem Jahr sammeln sich in der Innenjade größere Schwärme von metamorphisierten Jungheringen, auf die mit Ankerhamen, gelegentlich auch mit Granatkurren gefischt wird. Meist treten gleichzeitig Sprotten und einige zweijährige Heringe auf.

Im Herbst und Winter der Jahre 1948 bis 1952 und 1956 wurden in der Jade zur Untersuchung meristischer Merkmale einjährige Jungheringe, sog. „Spitzen“, gefangen. Konservierung und Färbung erfolgte in gleicher Weise wie bei den Heringslarven.

Insgesamt wurden die Wirbelzahlen bei fast viertausend Jungheringen bestimmt. In Tab. 4 sind die mittleren Wirbelzahlen für die einzelnen Jahrgänge aufgetragen.

Tabelle 4

Variation der Wirbelzahl bei Jungheringen aus der Innenjade

\begin{tabular}{ccccccccc} 
& Jahrg. & $\mathrm{n}$ & Vert.S & $\sigma^{2}$ & $\mathrm{~g} 1$ & $\mathrm{P}_{\mathrm{g} 1}$ & $\mathrm{~g} 2$ & $\mathrm{P}_{\mathrm{g} 2}$ \\
\hline ljährig & 1947 & 766 & 56.541 & 0,466 & $-0,132$ & $>0,05$ & $+0,382$ & $\sim 0,03$ \\
& 1948 & 661 & 56.555 & 0,450 & $-0,246$ & $<0,01$ & $+0,572$ & $<0,01$ \\
& 1949 & 593 & 56.578 & 0,403 & $-0,016$ & $>0,05$ & $+0,068$ & $>0,05$ \\
& 1950 & 525 & 56.551 & 0,404 & $-0,166$ & $>0,05$ & $+0,355$ & $\sim 0,05$ \\
& 1951 & 476 & 56.538 & 0,418 & $-0,280$ & $<0,01$ & $+1,008$ & $<0,01$ \\
& 1955 & 415 & 56.614 & 0,426 & $-0,038$ & $>0,05$ & $+0,111$ & $>0,05$ \\
& {$[1955$} & 271 & 56.561 & 0,595 & $-1,336$ & $<0,01$ & $+5,249$ & $<0,01]^{*}$ \\
2jährig & 1947 & 289 & 56.536 & 0,430 & $+0,013$ & $>0,05$ & $+0,664$ & $\sim 0,02$
\end{tabular}

*) Die Bestimmung der Wirbelzahl der 267 Spitzen von Nov. 1956 ergab bei 4 Tieren 53 Wirbelkörper, während in den vorhergehenden Jahren unter 3310 Spitzen kein Individuum und unter mehr als 15000 Heringslarven 1 Tier mit nur 53 Wirbeln angetroffen wurde. Im Gegensatz zu allen früheren Untersuchungen mußten die Wirbel der Spitzen im Dezember 1956 ungefärbt gezählt werden. Es kann nicht ausgeschlossen werden, daß dabei kleinere Verwachsungen in der Wirbelsäule übersehen wurden. Es wurden die statistischen Werte auch unter Fortlassung der 4 Extremvarianten berechnet. In Klammern befinden sich die Angaben für die Novemberprobe einschließlich der Tiere mit 53 Wirbeln.

Die Wirbelzahlen der "Spitzen“ in der Jade liegen höher als die mittleren Wirbelzahlen bei den Laichschwärmen auf der Doggerbank. Die hohen Werte können als Hinweis auf eine Mischpopulation gewertet werden. BERTELSEN u. Popp-MadSEN (1953-1957) fanden 1952 bis 1955 etwa die gleichen Wirbelsummen bei den 2-3jährigen Jungheringen der dänischen Ölheringsfischerei. In den voraufgegangenen Jahren 1949-51 hatte A. J. C. JENSEN (1950-52) zum Teil niedrigere Werte festgestellt. Aus Zusammenstellungen von SchUBERT (1957 a) für 1955 ergibt sich eine mittlere Wirbelzahl von 56.47. Auf die Bedeutung der Jungheringsschwärme der östlichen Nordsee für den Nachwuchs auf den einzelnen Laichgründen kann hier nicht eingegangen werden.

Ferner wurde geprüft, ob auch bei den Spitzen eine Beziehung zwischen Körpergröße und Wirbelzahl besteht. Während sich die Jahresmittel der Wirbelzahl nicht stark unterscheiden, bestehen zwischen den einzelnen Längen- 
gruppen der Spitzen eines Jahrganges bezüglich der Wirbelzahl mitunter deutliche Unterschiede (Tab. 5). Es ist möglich, daß die Beziehungen zwischen Wirbelzahl und Wachstumsgeschwindigkeit auch bei den Spitzen bestehenbleiben. Eine eingehende Analyse dieser Verhältnisse ist aber bei dem vorliegenden Jungheringsmaterial nicht möglich.

Schiefe und Exzeß der Variationskurve sind nur in 2 Jahren gesichert. Eine Diskussion der Annäherung des Verteilungsbildes der Wirbelzahl an die Normalverteilung wurde bereits früher gegeben (BüCKMANN 1950).

Tabelle 5

Wirbelzahl von Jungheringen verschiedener Größe

\begin{tabular}{|c|c|c|c|c|c|c|c|}
\hline Jahrgang & Fangtermin & $<89$ & $90-99$ & $100-109$ & $110-119$ & $120-29$ & $130-39 \mathrm{~mm}$ \\
\hline $\begin{array}{l}\mathbf{1 9 4 7} \\
\text { Vert. S. } \\
\text { n } \\
\sigma^{2}\end{array}$ & & $<100 \mathrm{~mm}=$ & $\begin{aligned}= & 56.557 \\
& 244 \\
& 0,560\end{aligned}$ & $>100 \mathrm{~mm}$ & $\begin{aligned}= & 56.534 \\
& 410 \\
& 0,422\end{aligned}$ & & \\
\hline $\begin{array}{l}1948 \\
\text { Vert. S. } \\
\mathrm{n} \\
\sigma^{2}\end{array}$ & 4. Nov. 1949 & $\begin{array}{l}56.57 \\
58 \\
0,53\end{array}$ & $\begin{array}{l}56.538 \\
288 \\
0,431\end{array}$ & $\begin{array}{l}56.585 \\
222 \\
0,488\end{array}$ & $\begin{array}{l}56.54 \\
78 \\
0,329\end{array}$ & $\begin{array}{l}56.47 \\
15 \\
0,7\end{array}$ & - \\
\hline $\begin{array}{l}1949 \\
\text { Vert. S. } \\
\mathrm{n} \\
\sigma^{2}\end{array}$ & Dez. 1950 & - & $\begin{array}{l}56.459 \\
135 \\
0,39\end{array}$ & $\begin{array}{l}56.595 \\
284 \\
0,383\end{array}$ & $\begin{array}{l}56.633 \\
139 \\
0,437\end{array}$ & $\begin{array}{l}56.68 \\
35 \\
0,40\end{array}$ & \\
\hline $\begin{array}{l}1950 \\
\text { Vert. S. } \\
\mathbf{n} \\
\sigma^{2}\end{array}$ & Dez. 1951 & - & - & & $\begin{array}{l}56.439 \\
157 \\
0,390\end{array}$ & $\begin{array}{l}56.58 .5 \\
313 \\
0,404\end{array}$ & $\begin{array}{l}56.66 \\
56 \\
0,409\end{array}$ \\
\hline $\begin{array}{c}1951 \\
\text { Vert. S. } \\
\mathrm{n} \\
\sigma^{2}\end{array}$ & Dez. 1952 & - & - & $\begin{array}{l}56.53 \\
34 \\
0,438\end{array}$ & $\begin{array}{l}56.521 \\
303 \\
0,403\end{array}$ & $\begin{array}{l}56.622 \\
111 \\
0,382\end{array}$ & $\begin{array}{l}56.40 \\
25 \\
0,50\end{array}$ \\
\hline $\begin{array}{c}\left.1955^{\circ}\right) \\
\text { Vert. S. } \\
\text { n } \\
\sigma^{2}\end{array}$ & $\begin{array}{r}\text { Nov. } 1956 / \\
\text { Jan. } 1957\end{array}$ & - & - & $\begin{array}{l}56.70 \\
77 \\
0,370\end{array}$ & $\begin{array}{l}56.624 \\
181 \\
0,436\end{array}$ & $\begin{array}{l}56.564 \\
156 \\
0,441\end{array}$ & \\
\hline
\end{tabular}

\%) Ohne die 4 Extremvarianten mit 53 Wirbeln, vgl. Fußnote zu Tab. 4.

Es sei noch darauf hingewiesen, daß ab 1951 bei etwa gleichem Fangtermin fast ausschließlich Spitzen über $10 \mathrm{~cm}$ Länge gefangen wurden, während in den vorhergehenden Jahren ein erheblicher Anteil der Proben kleiner als $10 \mathrm{~cm}$ war. Mehrere Autoren stellten fest, daß etwa vom gleichen Zeitpunkt an eine erhebliche Steigerung der Wachstumsgeschwindigkeit bei älteren Nordseeheringen erfolgte.

\section{f) Wirbelzahl der Larven und Wassertemperatur}

Wie für das Vorkriegsmaterial wurde auch für die Jahrgänge 1948-1951 eine negative Korrelation der Wirbelzahl und der winterlichen Wassertemperatur in der südlichen Nordsee festgestellt. Einen Uberblick über die Temperaturverhältnisse in den einzelnen Jahren lieferten die Synoptischen Monatskarten des Internationalen Rates für Meeresforschung. Als Grundlage 
der Berechnungen wurden die Oberflächentemperaturen beim Feuerschiff "S 2“ $\left(54^{0} 00^{\prime} \mathrm{N}, 3^{0} 32^{\prime} \mathrm{O}\right)$ gewählt (Dietrich 1956). In Abb. 5 sind die Wirbelsummen für die einzelnen Jahrgänge und die Temperaturmittel für November und Dezember der betreffenden Jahre dargestellt. Für den Monat November errechnet sich ein Korrelationskoeffizient $r=0,861 \pm 0,129$, für Dezember ist die Korrelation ebenfalls gesichert: $r=0,797 \pm 0,182$. Da bei Feuerschiff "S 2" die Wassersäule in diesen Monaten annähernd homotherm ist, geben die Oberflächenwerte einen guten Eindruck der Temperaturverhältnisse. Im Gebiet um „S 2" dürfte sich nach den früheren Untersuchungen ein Großteil der Doggerbank-Larven, die vorwiegend im Oktober bei ca. $12-13^{\circ} \mathrm{C}$ schlüpften, zu Beginn des Winters aufhalten. Die im Süden der Flämischen Bucht um diese Zeit schlüpfenden Heringslarven treffen höhere Temperaturen an. Die Kurven der November- bzw. Dezember-Mittel während des Untersuchungszeitraumes verlaufen dort aber gleichsinnig mit denen bei "S 2 ". So können die aus der Temperaturkorrelation zu ziehenden Schlüsse sowohl auf die Doggerbank-, als auch auf die Downslarven ausgedehnt werden. Auffallend ist das abweichende Verhalten des Jahrgangs 1952 (Abb. 5). Bei den ungewöhnlich niedrigen Wassertemperaturen wäre in diesem Jahr eine sehr hohe Wirbelzahl zu erwarten gewesen. Da diese nicht beobachtet wurde, bedeutet eine Einbeziehung dieses Jahrganges in die Korrelationsrechnung ein Verschwinden gesicherter negativer Korrelation zwischen Wassertemperatur und Wirbelzahl. Es läßt sich nicht feststellen, wodurch dies abweichende Verhalten 1952 bedingt war.

Zur Temperaturabhängigkeit der Wirbelzahl bei Clupea pallasii liegen Untersuchungen von Rounserell und Dahlgren (1932), Tester (1938) und Mc Hugh (1952) vor. Alle diese Arbeiten deuten darauf hin, daß die Wassertemperatur einen Einfluß auf die Anlage der Wirbelkörper durch Bestimmung der Segmentbildung während des embryonalen und frühlarvalen Lebens beim Hering besitzt. Ahnliches ist auch von anderen Fischen bekannt.

\section{g) Experimente zur Temperaturmodifikabilität der Wirbelzahl}

Bisher ist es nicht gelungen, Heringslarven mit einiger Sicherheit unter konstanten Bedingungen in größerer Zahl aufzuziehen, um die Temperaturmodifikabilität der Wirbelzahl im Experiment zu beweisen. Die Erbrütung der künstlich befruchteten Eier und die Hälterung der frischgeschlüpften Larven über einige Tage bietet keine methodischen Schwierigkeiten. Nach der Resorption des Dotters aber sterben regelmäßig alle oder fast alle Larven des Versuchs. In einer kurzen Mitteilung (HEMPEL 1953 a, dort weitere Literatur) ist über die Wiederaufnahme der vor dem Kriege auf Helgoland begonnenen Aufzuchtversuche berichtet worden. Bei diesen Experimenten im Max-PlanckInstitut für Meeresbiologie, Wilhelmshaven, wurde die Anzahl der Myomeren von Larven bestimmt. Nach Erbrütung bei verschiedenen Temperaturen zeigten die Nachkommen eines Elternpaares (Frühjahrslaicher aus der Elbe-Mündung) statistisch gesicherte Unterschiede in der Myomerenzahl. Die bei $13,4^{\circ} \mathrm{C}$ erbrüteten, frischgeschlüpften Larven wiesen im Mittel 56,56 $\pm 0,086$ Myomeren auf, die bei $8,8^{\circ} \mathrm{C}$ erbrüteten $57,10 \pm 0,099$ Myomeren (vgl. Tab. 1 bei Hempel 1953 a). Die Myomerenbildung scheint mit dem Verlassen des Eies noch nicht abgeschlossen und auch weiterhin temperaturabhängig zu sein. Eine 
Wiederholung dieser Versuche mit Brut von Doggerbanklaichern deutete darauf hin, daß unabhängig von der Temperatur diesen Larven eine höhere Myomerenzahl zukommt, entsprechend der höheren mittleren Wirbelzahl der Bankheringe gegenüber dem Küstenlaicher der Elbe (neuere Wirbelzählungen an Küstenheringen: Hempel 1953 b, Schubert 1957 b). Ahnliche Erbrütungsversuche unternahm Blaxter (1955). Nach freundlicher, schriftlicher und mündlicher Mitteilung fand er ebenfalls eine negative Covariation zwischen Myomerenzahl und Erbrütungstemperatur. Durch Verwendung geeigneter Untersuchungsmethoden gelang ihm dabei eine exaktere Bestimmung der Myomerenzahl im postanalen Abschnitt, als es uns in. Wilhelmshaven bei Untersuchung der lebenden Larve möglich war $\left.{ }^{6}\right)$. - Nach Fertigstellung der Aquarienanlagen auf Helgoland ist eine Fortsetzung dieser Arbeiten vorgesehen.

h) Diskussion zu den Untersuchungen über die Wirbelzahl der Heringslarven

Die früher mitgeteilten sowie die hier vorgelegten Untersuchungen ergaben Beziehungen zwischen der mittleren Wirbelzahl (Vert. S.) und 1. der mittleren Körpergröße ( $\left.\mathrm{L}_{\mathrm{abss}}\right)$ in einer Larvenp r ob e, 2. der relativen Körpergröße ( $\left.\mathrm{L}_{\text {rel }}\right)$ innerhalb einer Larveng r u p pe und 3. der Wassertemperatur zur Zeit der frühen Larvalentwicklung.

Als Erklärung für die festgestellte Beziehung Vert. $S . L_{a b b s}$ kann nicht das Einschieben zusätzlicher Wirbelanlagen in die Wirbelsäule mehr als $30 \mathrm{~mm}$ langer Larven angeführt werden. Die früher veröffentlichte Feststellung, daß eine solche Vermehrung der Wirbelanlagen beim Hering nie oder fast nie vorkommt, wurde in den hier vorgelegten sowic in den von Hamborf 1954 beschriebenen Untersuchungen bestätigt. Auf die Diskussion und Ablehnung anderer Erklärungsmöglichkeiten (vgl. BücKMANN 1950) soll hier nicht nochmals eingegangen werden $\left.{ }^{7}\right)$.

Als wahrscheinlichste Deutung der Ergebnisse bot sich die Annahme einer Selektion negativer Varianten der Wirbelzahl an. Diese Selektion geht, da zeitabhängig, dem Wachstum der Larven parallel. Mit Fortschreiten der Eliminierung der Minus-Varianten erhöht sich die mittlere Wirbelzahl im Verlaufe der Larvalentwicklung. Als Hinweis auf das Wirken einer solchen Selektion kann das Verschwinden von Schiefe und Exzeß, d. h. der Anhäufung negativer Varianten nach Abschluß des Larvenlebens gewertet werden. Bei den Spitzen wurden in der Regel keine gesicherten Abweichungen von der

6) Inzwischen veröffentlicht (BLAXTER 1957).

7) Einen neuen Hinweis auf die Abhängigkeit des Wachstums und der Wirbelzahl von den Umweltbedingungen während der ersten Lebenszeit glaubt Richardson noch bei adulten Heringen ablesen zu können. Zugleich soll die Körperlänge den Schlüpftermin innerhalb der Saison wiederspiegeln. Richardson (1956) untersuchte die Veränderungen der Wirbelzahl im Verlaufe der Fangsaison in der Whitby-Fischerei. Obschon im Mittel über die ganze Saison $L_{a b s}$ und Vert.S. der einzelnen Altersgruppen unabhängig variieren, finden sich bei Betrachtung der Aufeinanderfolge der Einzelproben gleichzeitige Anderungen beider Größen, die auf das Eintreffen von Heringsschwärmen hindeuten, dic - einheitlich im Alter - durch eine bestimmte mittlere Wirbelzahl und Körperlänge gekennzeichnet sind. RICHARDson nimmt an, daß diese Heringe jeweils e in e $m$ Laichakt entstammen und gemeinsam aufwuchsen. Unter Voraussetzung eincr negativen Korrelation zwischen Wirbelzahl und Erbrütungstemperatur läßt sich diese Erklärung nicht auf die in Abb. 3 betrachteten Doggerbanklarven anwenden. Die größten Larven mit der höchsten Wirbelzahl wären als die „Erstgeborenen" anzusprechen. Sie schlüpften aber auf der Doggerbank bei einer höheren Wassertemperatur als die spätergeborenen Larven. 
Normalverteilung festgestellt. Die Ausnahmen von dieser Regel können als Nachwirkung der Anomalien in der Variantenverteilung bei den Larven gewertet werden.

Die Abweichungen von der Beziehung Vert. S. / Labs im Nachkriegsmaterial lassen sich nicht ohne weiteres mit dieser "Selektionshypothese" vereinigen. Da die Abweichungen jeweils beim Auftreten einer neuen Larvengruppe zu beobachten sind, wäre in Erweiterung der "Selektionshypothese" daran zu denken, daß die Eliminierung in den einzelnen Larvengruppen bei einer bestimmten Körpergröße verschieden weit fortgeschritten ist, sei es, weil diese Gruppe einem stärkeren Selektionsdruck ausgesetzt war, oder weil sie eine andere mittlere Wachstumsgeschwindigkeit besaß.

Derartige Einflüsse können sich bei der beschränkten Anzahl von Proben, die wiederum auf wenigen "Larvenwellen“ fußen, bei den Jadeuntersuchungen stärker ausgewirkt haben als bei den weiträumigeren Vorkriegsarbeiten. Bei den Wirbeluntersuchungen der Vorkriegsjahre konnte nicht das ganze vorliegende Larvenmaterial systematisch bearbeitet werden. Durch Auswahl der Proben können manche Details verwischt worden sein. Leider gingen das Vorkriegsmaterial und die Urprotokolle durch Kriegseinwirkung verloren, so daß eine nachträgliche, detaillierte Prüfung unter den sich neu ergebenden Gesichtspunkten nicht möglich ist.

Es ist ebenfalls nicht festzustellen, ob auch im Vorkriegsmaterial eine Bindung der Wirbelzahl an die Wachstumsgeschwindigkeit vorlag. Für das Jahr 1953 vermochte die "Vorwüchserhypothese" (Vert. S./Labs) die genannten Abweichungen zu erklären. Es wird zu prüfen sein, ob dem allgemeinere Gültigkeit zukommt.

Es liegt nahe, an eine Kombination von "Selektions-" und „Vorwüchserhypothese" in folgender Weise zu denken: Es besteht eine genetische Beziehung zwischen Wachstumsgeschwindigkeit und Segmentierung des Körpers. Die Schlechtwüchsigkeit bedeutet einen Selektionsnachteil, da die kleineren, weniger beweglichen Larven leichter Feinden zum Opfer fallen. So kommt es zu einer Eliminierung der Minusvarianten von Wachstum und Wirbelzahl und zu einer Beziehung der Wirbelzahl zur absoluten und zur relativen Körpergröße. Da kein direkter Selektionsnachteil der niedrigen Wirbelzahl angenommen werden konnte, muß die "Selektionshypothese" ohnehin eine Koppelung dieses Merkmales mit anderen selektionistisch wichtigeren fordern. Sollte die Wachstumsgeschwindigkeit und die Wirbelzahl genetisch fixiert sein, müßten wir im Verlaufe der Generationen höhere Werte für Vert. S. erwarten, sofern nicht im weiteren Verlauf des Lebens der fördernde Einfluß der Selektion auf die Wirbelzahl durch andere Einflüsse kompensiert wird. Unter Vermeidung der Annahme einer genetischen Fixierung könnte man vermuten, daß Wirbelzahl und Wachstumsgeschwindigkeit während des frühen Larvenlebens bestimmt werden, indem die Larven eines Laichgebietes infolge wechselnder Transportbedingungen unterschiedlichen Temperatur- und Nahrungsverhältnissen ausgesetzt sind.

Die vorliegenden Ergebnisse zeigen, daß die Wirbelzahl einer Heringspopulation während der Larven- und ersten Jugendzeit Veränderungen unterworfen ist. Welcher Bedeutung daneben der Erbmasse der einzelnen Larvengruppen für die Ausbildung der endgültigen mittleren Wirbelzahl zukommt, läßt sich nicht entscheiden. Auch können die Abhängigkeit der Wirbelzahl von der Wassertemperatur, die Selektion und die Beziehung zur Wachstumsgeschwindigkeit in ihrem Einfluß auf die Wirbelsumme nicht gegeneinander abgewogen werden. 
Der Rückschluß auf die Herkunft einer Larvenprobe auf Grund eines Vergleichs ihrer Vert. S. mit der in Frage kommenden Elternpopulation ist durch die Beziehung Vert. S./Lahs zumindest sehr erschwert. Eine weitere Behinderung tritt auf, wenn nicht sichergestellt ist, daß diese Probe die betreffende Larvengruppe gleichmäßig erfaßt, da dann die Bindung Vert. S./Lre! ins Gewicht fällt.

Nach dem Stande unserer derzeitigen Kenntnisse bietet sich uns folgende Vorstellung von der Entwicklung der mittleren Wirbelzahl einer Larvengruppe bis zur Metamorphose an: Genetisch wird eine Reaktionsnorm für die durch endogene und exogene Einflüsse bestimmte Wirbelzahl festgelegt. Diese Reaktionsnorm dürfte für die 3 großen Heringsrassen des Ostatlantik: Atlantoskandischer Hering, Herbstlaicher der Nordsee und Frühjahrslaicher der Küstengebiete verschieden sein ( vgl. BLAXTER 1956). Vielleicht bestehen geringere Unterschiede auch zwischen den einzelnen, weniger isolierten Laichgemeinschaften. Der Umweltfaktor Wassertemperatur scheint während der ersten Lebenszeit bedeutungsvoll für die spätere Ausbildung der Wirbelsäule zu sein. Im weiteren Leben der Larvengruppe kommt es durch Selektionsvorgänge zu einer Erhöhung der Wirbelzahl. Durch eine Beziehung zwischen Wirbelzahl und Wachstumsgeschwindigkeit ist zumindest zeitweilig bei der Wanderung zur Küste eine räumliche Staffelung einer Larvengruppe in "Larvenwellen " mit unterschiedlicher Körpergröße, damit verschiedener Schwimmleistung und mit verschiedener Körpergröße möglich. Dies dürfte von biologischer Bedeutung für Wachstumsgeschwindigkeit und Selektion sein. Eine küstenwärts gerichtete Längenstaffelung in der Larvenbevölkerung wurde bereits früher nachgewiesen (BÜCKMANN 1950).

Die Jungheringe der Deutschen Bucht und der südöstlichen Nordsee insgesamt stellen ein Gemisch von Tieren verschiedener Herkunft dar, das mit unseren herkömmlichen Methoden nicht befriedigend analysiert werden kann. So ist über die Vermischungs- und Selektionsvorgänge zwischen der Metamorphose und dem Auftreten als Erstlaicher auf den verschiedenen Laichplätzen wenig bekannt. Ebenso ist ungeklärt, wie sich die Jungheringbestände bei Erlangung der Geschlechtsreife auf die verschiedenen Vorlaich- und Laichgemeinschaften verteilen. Diese Faktoren bestimmen aber die mittlere Wirbelzahl bei den Erstlaichern.

Für die Veränderungen der mittleren Wirbelzahl eines Jahrganges liefert die Zahlentafel 7 bei FRIDRIKsson 1956 wertvolle Unterlagen. Es handelt sich dabei um Heringe des "Northern Norwegian Type“, die unter Island gefangen wurden. Im Verlaufe der Jahre steigt die Wirbelzahl innerhalb eines Jahrganges sukzessive an. Auch hier mögen Selektionsvorgänge oder ein unterschiedliches Verhalten von Heringen verschiedener Wirbelzahl bei der Wahl des Laichplatzes eine Rolle spielen. Abrupte Anderungen in der Laichplatzwahl bei einem bestimmten Lebensalter konnte Wood (1956) für Heringe nordschottischer Laichplätze nachweisen.

Die von Z IJLSTRA (1956) angegebenen Wirbelzahlen deuten darauf hin, daß die Angehörigen eines Jahrganges auf einem Laichplatz in aufeinanderfolgenden Jahren nicht mit der gleichen mittleren Wirbelzahl auftreten. So scheint es nicht erwiesen, daß ein Hering im Laufe seines Lebens immer wieder den gleichen Laichplatz aufsucht. Der $z$. T. geringe Probenumfang schließt aber in diesem Fall die Gefahr von Fehldeutungen ein. Die Untersuchungen von Richardson 1956 zeigten klar die großen Schwankungen, denen der Auf- 
bau eines Heringsvorkommens auch bezüglich der meristischen Merkmale innerhalb einer Saison unterliegt.

\section{Zusammenfassung}

In Fortsetzung der Untersuchungen der Biologischen Anstalt Helgoland über die Heringsbrut in der südlichen Nordsee wurden in den Jahren 1948 bis 1953 Verbreitung, Längenzusammensetzung und Wirbelzahlen der $\mathrm{He}$ ringslarven des Jadegebietes studiert.

1. Von Februar bis Mai dringen schubweise Heringslarven („Larvenwellen") unterschiedlicher Körperlänge in die Jade ein.

2. An Hand der Längenzusammensetzung der Larvenbevölkerung in den einzelnen Monaten lassen sich mehrere Larvengruppen unterscheiden und in den Kurvenbildern verschiedener Jahre (Abb. 1) wiedererkennen. Mit Hilfe älterer und neuer Untersuchungen in See können diese Larvengruppen drei Laichgebieten herkunftsmäßig zugeordnet werden: 1. dem sommerlichen Laichen in der mittleren Nordsee; 2. dem Herbstlaichen im Doggerbankgebiet; 3. dem Frühwinterlaichen in der südlichen Flämischen Bucht; gelegentlich wurden auch noch jüngere Larven festgestellt. Ihre Herkunft von den Laichplätzen im östlichen Kanal war zumindest 1953 fraglich.

3. Die statistische Verteilung der Wirbelzahlen bei den Heringslarven folgt nicht der Normalverteilung, sondern ist statistisch gesichert schief und exzessiv. Bei einjährigen Jungheringen treten solche Abweichungen nur noch gelegentlich auf.

4. Neben einer Korrelation zwischen mittlerer Wirbelzahl und mittlerer absoluter Körpergröße (Vert. S./Labs) zeigte sich für den Jahrgang 1952 eine Beziehung zwischen Wirbelzahl und Wachstumsgeschwindigkeit, indem innerhalb einer bestimmten Larvengruppe die größten Larven die höchste Wirbelzahl aufwiesen.

5. Es kann nicht entschieden werden, ob die hohen Wirbelzahlen kleiner Larven im April und Mai 1952 und 1953, die als Downslarven angesprochen werden, durch deren Herkunft bedingt sind. Die Möglichkeit muß offenbleiben, daß die Wirbelzahlen so hoch liegen, weil es sich bei den untersuchten Larven um die Vorwüchser der betreffenden Larvengruppe (Downslarven) handelt.

6. Die Wirbelzahl der in den Nachkriegsjahren untersuchten Heringslarven liegt statistisch gesichert über den Mittelwerten in den Jahrgängen 1928-35. Zum Teil kann dies durch Unterschiede in der Längenzusammensetzung erklärt werden.

7. In der Jade gefangene Jungheringe (meist einjährig), der Jahrgänge 1947 bis 1951 und 1955 wiesen eine etwas höhere Wirbelzahl auf als die Larven. In einzelnen Jahren zeigte sich auch hier eine Abhängigkeit der Wirbelzahl von der Körperlänge. Seit 1951 lagen die mittleren Körperlängen der einjährigen Heringe über den Werten der früheren Jahre.

8. Auch für die Nachkriegsjahrgänge der Heringslarven besteht eine negative Korrelation zwischen Wirbelzahl und Wassertemperatur im November und Dezember in der südlichen Nordsee.

9. Die Erbrütung künstlich befruchteter Eier bei verschiedenen Temperaturen ergab eine negative Korrelation zwischen der Erbrütungstemperatur und 
der Myomerenzahl frischgeschlüpfter Larven. Auch nach dem Schlüpfen scheint die Anzahl der sichtbaren Myomeren noch temperaturabhängig zu steigen. In den noch nicht abgeschlossenen Versuchen fanden wir Anzeichen dafür, daß Bankheringslarven eine höhere Myomerenzahl als bei gleicher Temperatur erbrütete Küstenheringe aufweisen. Ein genetischer Einfluß kann also nicht ausgeschlossen werden.

10. Auf Grund der erzielten Ergebnisse und älterer Untersuchungen wird diskutiert, welchen Veränderungen die mittlere Wirbelzahl einer Larvengruppe unterworfen ist. Die mittlere Wirbelzahl eines Laichschwarmes dürfte auch von Selektions- und Vermischungsvorgängen abhängig sein, die noch nach der Metamorphose wirken.

\section{Summary}

In continuation of earlier investigations (BüCKMANN 1950) on the distribution of Herring fry in the German Bight special observations in the Jadeestuary were carried out from 1948 to 1953. Catches were made with Petersen's young fish trawl once or twice per month at $5-7$ stations in the area. Herring larvae of different length distribution arrive the Jade in several waves from February to May.

I. On the grounds of their modal length 4 larvae groups may be discerned. In comparision with the observations in pre-war and post-war years in the open sea it is possible to determine the presumable origin of these larvae groups:

1. Larvae arising from the summer spawning in the western part of the Middle North Sea were found in the Jade in February and March with a mean length of about $40 \mathrm{mms}$.

2. The smaller larvae of $30-35 \mathrm{mms}$, which presumable had hatched in the autumn in the Doggerbank, arrived in the inshore area in March.

3. Two groups of different size („ältere" and „jüngere Downslarven“) may be derived from the spawning places of the southern Flemish Bight. The larger ones may be spawned in November-December. Their mean length is at the time of arrival in the Jade in April or May of about 30 to $35 \mathrm{mms}$. The offspring of the January spawning in the eastern Channel came only in single years at the end of April or in May into the Jade. Then they were about $25 \mathrm{mms}$ long.

II. The number of vertebrae of about 5000 larvae has been counted:

1. The distribution of the vertebral number doesn't follow the normal distribution as in samples of adult herring. The variation curve is skew and peaked. Only in few cases we found a similar distribution in samples of 1 year old herring.

2. As in pre-war years a correlation was established between the mean number of vertebrae and the mean length of larvae. In the year class 1952 there was also a relation between vertebral number und growth rate: In a particular larvae group, presumably of uniform origin, the largest and probably faster growing individuals showed a higher average than do the smaller ones.

3. A preliminary hypothesis is given on account of these results: During 
the larval development, a part of the larvae with low vertebral number is eliminated from the group. Conceivably these are the variants of low growth. That results in an increase of the average number of vertebrae of the group. At the same time the degree of deviation from distribution of vertebrae counts decreases.

4. In consequence of these variations and changes of Vert. S. inside a given larval group it is rather difficult to separate larval groups by their vertebral number. Partly the distinct difference found between the average numbers of vertebrae of pre-war and post-war samples may be caused by differences in the length composition.

5. The determination of Vert. S. of whitebait in the Jade showed a slightly higher Vert. S. for the older herring than for the larvae of the same year class. Only in some years a relation between length and Vert. S. is evident also in 1 year old herring. It may be worth mentioning that the mean length of the whitebait was higher after 1950 than in the corresponding month of the previous years.

6. The number of vertebrae is inverselly correlated to the water temperature during the first months of larval life (November, December).

7. Rearing experiments showed a similar negative correlation between the rearing temperature and the number of myotomes of yolk sac larvae. There seems to be also a temperature dependence of the myotome count after hatching.

8. Our observations led us to the assumption that Vert. S. and, probably, other meristic characters as well are subject to modifications which are assumed to occur only within limits fixed by heredity. The mean number of vertebrae in a sample of a given larval group may be the result of interactions of inheritance and modifications caused directly by environmental factors. Moreover selection processes and distribution of the larvae in space and time are supposed to influence the average values. After metamorphosis the selection combined with mixing processes and, on the other hand, the separating in different groups may continue. So the Vert. S. of spawning shoals are assumed to be caused by a complicated system of influences. Only some of these influences are known until now.

\section{Literaturverzeichnis}

Bertelsen, E, und K. Popp-Madsen, 1953-1957: Young Herring from the Bløden Ground Area. Cons. Perm. Internat. Expl. Mer, Ann. Biol. 9 (1952), 179, 180; 10 (1953), $155 / 56 ; 11(1954), 125 / 27 ; 12(1955)$.

Blaxter, J. H. S., 1955: Herring Rearing I. The Storage of Herring Gametes. Mar. Res., Scott. Home Dep. 1955, 3.

- 1956: Herring Rearing II. The Effect of Temperature and other Factors on Development. ibid. 1956, 5 .

- 1957: Herring Rearing III. The Effect of Temperature and other Factors on Myotome Counts. ibid. 1957, 1.

Bohl, H. J., 1954: (Herring:) Larvae in the Southern North Sea and the German Bight during March 1953. Cons. Perm. Internat. Explor. Mer, Ann. Biol. 10 (1953), 151-52.

Buchanan-Wollaston, H. J., 1933: Some Modern Statistical Methods: their Application to the Solution of Herring Race Problems. Cons. Perm. Internat. Expl. Mer, J. du Conseil 8, 7-47.

B ü ckmann, A., 1953: Fischbrut u. Evertebraten-Großplankton auf der Fahrt der "Gauss" im Febr./März 1952. Ber. Dtsch. Wiss. Komm. Meeresforsch. 13, 154-174. 
- 1950/51: Die Untersuchungen der Biologischen Anstalt über die Okologie der Heringsbrut in der südlichen Nordsee. I. Helgol. Wiss. Meeresunters. 3, 1-57; - II, ibid. 3, 172-205.

Bü ckmann, A. u. G. Hempe 1, 1953: Beobachtungen über Verteilung und Entwicklungszustand der Heringslarven an der west- u. ostfriesischen Küste im Frühjahr 1952. Kurzc Mitteil. aus d. fischereibiolog. Abt. d. Max-Planck-Inst. f. Meeresbiolog., Wilhelmshaven 2, 24-38.

- 1956: Ms. Some Results of German Investigations on Herring Larvae. Cons. Perm. Internat. Explor. Mer, Special Scient. Meeting on Herring Races Nr. 1.

Conseil permanent pour l'exploration de la mer, Service hydrographique: Synoptische Monatskarten.

Dietrich, G., 1953: Verteilung, Ausbreitung und Vermischung der Wasserkörper in der südwestlichen Nordsee auf Grund der Ergebnisse der "Gauss"-Fahrt im Februar/März 1952. Ber. Dtsch. Wiss. Komm. Meeresforsch. 13, 104-129.

- 1956: Hydrography of the German Bight. Cons. Perm. Internat. Explor. Mer, Ann. Biol. 9 (1954), 43-45.

Fridrikss on, A., 1956, Ms: The Tribes in the North-Coast Herring of Iceland with Special Reference to the Period 1948-1955. Cons. Perm. Internat. Explor. Mer, Special Scient. Meeting on Herring Races. Nr. 8.

Gillbricht, M., 1956: Die Hydrographie des Jadebusens und der Innenjade. Veröff. Inst. Meereskunde Bremerhaven. 4, 153-170.

Goulden, C. H., 1952: Methods of Statistical Analysis. 2nd Ed. New York, London.

H a md o r f, K., 1954: Der Entwidklungszustand der Heringslarven als Unterscheidungsmerkmal bei der Populationsanalyse im ostfriesischen Küstenbereich. Diplomarbeit. Math.-Nat. Fakultät, Univ. Hamburg.

- 1956: Uber die Verwendbarkeit der Schwanzwirbelverknödierung der Heringslarven als Unterscheidungsmerkmal bei der Populationsanalyse. Kurze Mitt. Inst. Fischereibiol. Univ. Hamburg 6, 10-21.

Hempe1, G, 1953: Meristische Merkmale der Frühjahrsheringe in Ems und Elbe. Kurze Mittl. Fischereibiol. Abt. Max-Planck-Inst. Meeresbiologie, Wilhelmshaven 3, 43-45.

- 1953: Die Temperaturabhängigkeit der Myomerenzahl beim Hering (Clupea harengus L.). Naturwissenschaften 40, 46-48.

- 1957: (Herring:) Larvae in the Southern North Sea during March 1955 and 1956. Cons. Perm. Internat. Explor. Mer, Ann. Biol. 13 (1956), im Druck.

Jensen, A. J. C., 1950-51: Young Herring off Esbjerg. Cons. Perm. Internat. Explor. Mer, Ann. Biol. (1949) 6, 173/4; (1950) 7, 128.

- 1952: Young Herring at the Bladen Ground-Clay Deep. Cons. Perm. Internat. Explor. Mer, Ann. Biol. (1951) 8, 142/3.

Mc Hugh, J. L., 1942: Vertebral Number of Young Herring in Southern British Columbia. J. Fish. Res. Bd. Canad. 5, 474-84.

Richardson, I. D., 1956. Ms: Selection of Representative Samples in a Herring Fishery. Cons. Perm. Internat. Explor. Mer, Comparative Fishing Comm. Nr. 58.

Rounsefell, G. A. u. E. A. Dahlgren, 1932: Fluctuations in the Supply of Herring, Clupea pallasii, in Prince William Sound, Alaska, Bull. U.S. Bur. Fish. 47, 263/91.

Schubert, K., 1957: Survey of the German Commercial Herring Fisheries and the Biological Conditions of the. Stocks. Cons. Perm. Internat. Explor. Mer, Ann. Biol. 12 (1955).

- 1957: Coastal Herring from the Mouth of the River Elbe. Cons. Perm. Internat. Explor. Mer, Ann. Biol. 12 (1955).

Tesch, J. J., 1987: Racial Investigations on Herring Larvae in the South Western Inlets of Holland. Cons. Perm. Internat. Explor. Mer, Rapp. et Verb. 105, 3, S. 7.

Tester, A. L., 1937: Populations of Herring (Clupea pallasii) in the Coastal Waters of British Columbia. J. Biol. Board Canada, 3, 108/143.

- 1938: Variation in the mean Vertebral Count of Herring (Clupea pallasii) with Water Temperature. Cons. Perm. Int. Expl. Mer, J. du Conseil, 13, 71-75.

- 1949: Populations of Herring along the West Coast of Vancouver Island on the Basis of mean Vertebral Number, with a Critique of the Method. J. Fishery Res. Bd. Canada, 7, $7 ; 403-20$.

Wood, H., 1956, Ms: Race Characteristics of the Herring on the Clyde Estuary. Cons. Perm. Internat. Explor. Mer, Special Scient. Meeting on Herring Races. Nr. 19.

Zijlstra, J. J., 1956, Ms: On the Spawning Communities of the Herring in the Southern North Sea and the English Channel (Preliminary Results). Cons. Perm. Internat. Explor. Mer, Special Scient. Meeting on Herring Races, Nr. 15. 